\title{
Earth-based detection of the millimetric thermal emission from the nucleus of comet 8P/Tuttle ${ }^{\star}$
}

\author{
J. Boissier ${ }^{1,2,3}$, O. Groussin ${ }^{4}$, L. Jorda ${ }^{4}$, P. Lamy ${ }^{4}$, D. Bockelée-Morvan ${ }^{5}$, \\ J. Crovisier ${ }^{5}$, N. Biver ${ }^{5}$, P. Colom ${ }^{5}$, E. Lellouch ${ }^{5}$, and R. Moreno ${ }^{5}$ \\ ${ }^{1}$ Istituto di Radioastronomia - INAF, via Gobetti 101, Bologna, Italy \\ e-mail: boissier@ira.inaf.it \\ 2 ESO, Karl Schwarzschild Str. 2, 85748 Garching bei Muenchen, Germany \\ 3 Institut de radioastronomie millimétrique, 300 rue de la piscine, Domaine universitaire, 38406 Saint Martin d'Hères, France \\ ${ }^{4}$ Laboratoire d'Astrophysique de Marseille, Université de Provence, CNRS, 38 rue Frédéric Joliot-Curie, 13388 Marseille Cedex 13 , \\ France \\ ${ }^{5}$ LESIA, Observatoire de Paris, 5 place Jules Janssen, 92195 Meudon, France
}

Received 3 November 2010 / Accepted 17 January 2011

\section{ABSTRACT}

\begin{abstract}
Context. Little is known about the physical properties of cometary nuclei. Measuring the thermal emission of a nucleus is one of the few means of deriving its size, independently of its albedo, and constraining some of its thermal properties. This emission is difficult to detect from Earth but space telescopes, such as th Infrared Space Observatory, Spitzer Space Telescope, and Herschel Space Observatory, allow reliable measurements to be made in the infrared and the sub-millimetre domains.

Aims. We attempt to characterize more accurately the thermal properties of the nucleus of comet 8P/Tuttle using multiwavelength space- and ground-based observations, in the visible, infrared, and millimetre range.

Methods. We used the Plateau de Bure Interferometer to measure the millimetre thermal emission of comet $8 \mathrm{P} / \mathrm{Tuttle}$ at $240 \mathrm{GHz}$ $(1.25 \mathrm{~mm})$ and analysed the observations with the shape model derived from Hubble Space Telescope observations and the nucleus size derived from Spitzer Space Telescope observations.

Results. We report on the first detection of the millimetre thermal emission of a cometary nucleus since comet C/1995 O1 Hale-Bopp in 1997. Using the two contact-sphere shape model derived from Hubble Space Telescope observations, we constrain the thermal properties of the nucleus. Our millimetre observations are most accurately reproduced by assuming: i) a thermal inertia lower than $\sim 10 \mathrm{~J} \mathrm{~K}^{-1} \mathrm{~m}^{-2} \mathrm{~s}^{-1 / 2}$, and ii) an emissivity lower than 0.8 , which is indicative of a non-negligible contribution of the colder sub-surface layers to the outcoming millimetre flux.
\end{abstract}

Key words. comets: individual: $8 \mathrm{P} /$ Tuttle - radio continuum: planetary systems - techniques: interferometric

\section{Introduction}

Much of the scientific interest in comets stems from their potential role in elucidating the processes responsible for the formation and evolution of the Solar System. They appeared in the outer regions of the protoplanetary disk, when the giant planets were formed by core accretion (Safronov \& Zvjagina 1969; Pollack et al. 1996) or disk instability (Cameron 1978; Boss 2003). In addition to the asteroids, Centaurs, and transneptunian objects, comets are the remnants of the planetesimals that were not accumulated into the planets. Comets formed in the giant planet region were ejected into the outer Solar System to form the Oort Cloud. Some of them returned to the inner Solar System as long-period comets (now called nearly isotropic comets (NIC), Duncan et al. 1988), such as C/1995 O1 HaleBopp, or became periodic comets after several perihelion passages their orbits being shortened by gravitational perturbations. The later set of comets constitute the Halley-family comets (or returning NIC) to which $1 \mathrm{P} /$ Halley and $109 \mathrm{P} /$ Swift-Tuttle are representatives. Comet $8 \mathrm{P} /$ Tuttle also belongs to this group: its

* Based on observations carried out with the IRAM Plateau de Bure Interferometer. IRAM is supported by INSU/CNRS (France), MPG (Germany) and IGN (Spain). orbit has a high ecliptical inclination $\left(55^{\circ}\right)$ with a short period (13.5 years). On the other hand, it is believed that the Kuiper Belt, gravitationally influenced by the outer planets, supplied the population of Jupiter-family short-period comets (now called ecliptic comets EC, Duncan et al. 1988). Comets 19P/Borrelly and 9P/Tempel 1, investigated by the Deep Space 1 and Deep Impact missions, respectively, are typical comets of this category.

Given their different origins and the different evolutionary paths followed since their formation, one could expect to observe different physical properties for the NICs and ECs. For the time being, no obvious correlation between the chemical and physical properties of a comet and its dynamical class has been measured (Crovisier et al. 2009).

One of the possible ways to investigate differences between the two classes of comets is to measure their size distribution. Reliable size determinations have been obtained for only 13 NICs (Lamy et al. 2004), using ground- and space-based telescopes in the visible and infrared domain. The range of radii is surprisingly broad, from 0.4 to $37 \mathrm{~km}$, much broader than that of the ECs but with only 13 objects, robust conclusions cannot be drawn. Further measurements are required to confirm this trend. In this context, it is important to develop new methods 
to obtain reliable size estimates from the Earth. The millimetre wavelength range is well suited for this purpose because the atmospheric transmission is better than in the infrared, and also because the ALMA observatory will offer unique observing capabilities in the near future. However, the flux of a comet nucleus is much lower in the millimetre domain than in the infrared, and observations are still challenging and reserved to a few bright comets. Whenever available, we find that the various data sets at different wavelengths (visible, infrared, millimetre) complement each other and allow detailed studies of the nucleus properties.

Comet 8P/Tuttle was observed in 1992 using the Nordic Optical Telescope (NOT) at 6.3 AU from the Sun and appeared inactive at this time. From its apparent magnitude, the nucleus diameter was estimated to be $15.6 \mathrm{~km}$ (Licandro et al. 2000), but photographic observations performed in 1980 at 2.3 AU measured a nucleus three times smaller or highly elongated. The close approach to the Earth in December 2007January 2008 ( $0.25 \mathrm{AU})$ and its then supposedly large size made it a very interesting target for Earth-based observations. In 2007-2008, the comet was observed with different techniques in the visible (lightcurve measurements with the Hubble Space Telescope (HST), Lamy et al., in prep.), the infrared (Spitzer Space Telescope (SST) observations, Groussin et al. 2008, and Groussin et al., in prep.), and the radio domains (radar experiments with Arecibo, Harmon et al. 2010). To complement this multiwavelength set of observations, we observed comet 8P/Tuttle with the Plateau de Bure Interferometer to measure the millimetre thermal emission of its nucleus. The only similar measurement of a comet nucleus was performed more than ten years ago, in 1997 for comet Hale-Bopp (Altenhoff et al. 1999), which demonstrates the difficutly of these observations and the unique opportunity offered by comet 8P/Tuttle.

In this paper, we present the results of our observations of comet 8P/Tuttle carried out with the Plateau de Bure interferometer at $1.25 \mathrm{~mm}(240 \mathrm{GHz})$ in December 2007-January 2008. A description of the observations is given in Sect. 2. We analysed the data using a shape model of Lamy et al. (in prep.) and a thermal model of the nucleus, which are described in Sect. 3. The results are presented in Sect. 4 and summarized in Sect. 5.

\section{Observations}

Comet 8P/Tuttle was observed twice at a wavelength of $1.25 \mathrm{~mm}$ $(240 \mathrm{GHz})$ with the IRAM interferometer on 29 December 2007 and 8 January 2008 (Table 1). The IRAM interferometer is a six antennas (15 m each) array located at the Plateau de Bure, in the French Alps, and equipped with heterodyne, dualpolarization, receivers operating around 1,2 , and $3 \mathrm{~mm}(230$, 150 , and $100 \mathrm{GHz}$, respectively). On both observing dates, the array was set in a compact configuration with baseline lengths ranging from $\sim 20$ to $150 \mathrm{~m}$. This results in a synthesized beam diameter of about $1.2^{\prime \prime}$ at $240 \mathrm{GHz}$. The entire calibration process was performed using the GILDAS software packages developed by IRAM (Pety 2005).

December observations: For the observations acquired on 29 Dec. 2007, the comet was tracked using an ephemeris computed using the JPL solution K074/19 for its orbital elements. The instrument was tuned in a way to search for $\mathrm{CH}_{3} \mathrm{OH}$ lines around $241 \mathrm{GHz}$, four narrow correlator units being dedicated to the $\mathrm{CH}_{3} \mathrm{OH}$ lines, and a total bandwidth of $1.2 \mathrm{GHz}$ being used to measure the continuum emission of the comet. We observed $8 \mathrm{P} /$ Tuttle from $15 \mathrm{~h}$ to $23 \mathrm{~h} \mathrm{UT}$, under decent conditions (system temperature of $200 \mathrm{~K}$ and a phase rms of $\sim 30^{\circ}$ ) during the first half, until $19 \mathrm{~h}$ UT. The second half of the observations were affected by poor conditions (unstable phase, cloudy weather) and these data are unusable. The final dataset represents $\sim 1.6 \mathrm{~h}$ of on-source data aquired between $15 \mathrm{~h}$ UT and $19 \mathrm{~h} \mathrm{UT}$. The gain calibration sources (observed every $22 \mathrm{~min}$ to monitor the instrumental and atmospheric phase and amplitude variations) were $0133+476$ and $0059+581$. MWC349 was used to determine the absolute flux scale. The pointing and focus corrections were measured every 40 and $80 \mathrm{~min}$, respectively. Some single dish spectra of the $\mathrm{CH}_{3} \mathrm{OH}$ lines were recorded throughout the observing period. The sky contribution was cancelled in position switching mode (ON-OFF), with an OFF position at a distance of $5^{\prime}$ from the comet. We do not retrieve any $\mathrm{CH}_{3} \mathrm{OH}$ line detection in interferometric mode but the lines were detected in the $\mathrm{ON}-\mathrm{OFF}$ spectra. Their analysis is presented in Biver et al. (2008) with other single dish observations of 8P/Tuttle.

Given the distance of the comet, the apparent diameter of a nucleus with a radius of $10 \mathrm{~km}$ is about $0.1^{\prime \prime}$ and we do not expect to resolve the nucleus of $8 \mathrm{P} /$ Tuttle in the Plateau de Bure data. As a result, we analysed the continuum observations in the Fourier plane, fitting the Fourier transform (FT) of a point source to the observed visibilities. We measured a flux $F=2.4 \pm 0.7 \mathrm{mJy}$ located at an offset of $\left(-0.5^{\prime \prime},-1.4^{\prime \prime}\right)$ in (RA, Dec) with respect to the pointed position (the astrometric precision, estimated by dividing the beam size by the signal-tonoise ratio, is $\left.\sim 0.3^{\prime \prime}\right)$. Compared to the latest ephemeris solution (JPL K074/27, including observations performed up to October 2008), the offset is $\left(0.4^{\prime \prime}, 3.8^{\prime \prime}\right)$. To illustrate the result of this fit, we present in Fig. 1 the real part of the visibilities as a function of the uv-radius. The visibility table has been shifted to the position where the point source was found in the fit, so that the imaginary part of the visibilities is null and their real part is the source flux. In the figure, the real part of the visibilities has been averaged in $30 \mathrm{~m}$ bins in uv-radius. We overplot the point source flux found by the fitting procedure. The absolute flux calibration adds an uncertainty of $20 \%$ in both the flux and its uncertainty. We estimate the overall $\pm 1 \sigma$ limits as $F^{+1 \sigma}=1.2 \times(F+1 \sigma)=3.7 \mathrm{mJy}$ and $F^{-1 \sigma}=0.8 \times(F-1 \sigma)=1.4 \mathrm{mJy}$. From this, we deduce the final flux and corresponding errorbars $F=2.4_{-1.0}^{+1.3} \mathrm{mJy}$.

January observations: An updated solution of orbital elements (JPL K074/21) was used to compute the ephemeris of the comet the 08 Jan. 2008. Our single dish observations of the comet at the IRAM-30 m telescope (in early January, Biver et al. 2008) showed that its gas production was insufficient to enable interferometric study of the coma. As a result, we dedicated all the correlator units of the Plateau de Bure to continuum observations for this second run, without changing the observing frequency $240 \mathrm{GHz}$. This results in a bandwidth of $\sim 2 \mathrm{GHz}$, which represents a gain of a factor 1.3 in point source sensitivity with respect to the December observations. The comet was observed from $15 \mathrm{~h}$ UT to $21 \mathrm{~h} \mathrm{UT}$, under poor (degrading with time) phase stability conditions (a system temperature of $200 \mathrm{~K}$ and a phase rms of $\sim 30-100^{\circ}$, depending on the baseline length). As a result, we use in the analysis only the first two hours of observations ( $1.3 \mathrm{~h}$ on source), when the conditions were the best. Although the integration time is longer for the December observing run, the larger bandwidth of the January data results in a lower thermal noise for this dataset. The gain calibration sources 
Table 1. Log of the Plateau de Bure observations of the comet 8P/Tuttle.

\begin{tabular}{lcccccc}
\hline \hline Date & $\begin{array}{c}\mathrm{UT} \\
\mathrm{h}\end{array}$ & Ephemeris & $\begin{array}{c}\Delta \\
\mathrm{AU}\end{array}$ & $\begin{array}{c}r_{\mathrm{h}} \\
\mathrm{AU}\end{array}$ & $\phi^{a}$ & $\begin{array}{c}\text { Flux }^{b} \\
\mathrm{mJy}^{2}\end{array}$ \\
\hline 29 Dec. 2007 & $15-19$ & JPL K074/19 & 0.26 & 1.11 & $54^{\circ}$ & $2.4 \pm 0.7$ \\
08 Jan. 2008 & $15-17$ & JPL K074/21 & 0.28 & 1.06 & $65^{\circ}$ & $3.0 \pm 0.5$ \\
\hline
\end{tabular}

Notes. ${ }^{(a)}$ Phase angle of the observations. ${ }^{(b)}$ The flux and its uncertainty are measured by a fit of a point source to the observed visibilities. The absolute flux calibration adds an uncertainty of $20 \%$.
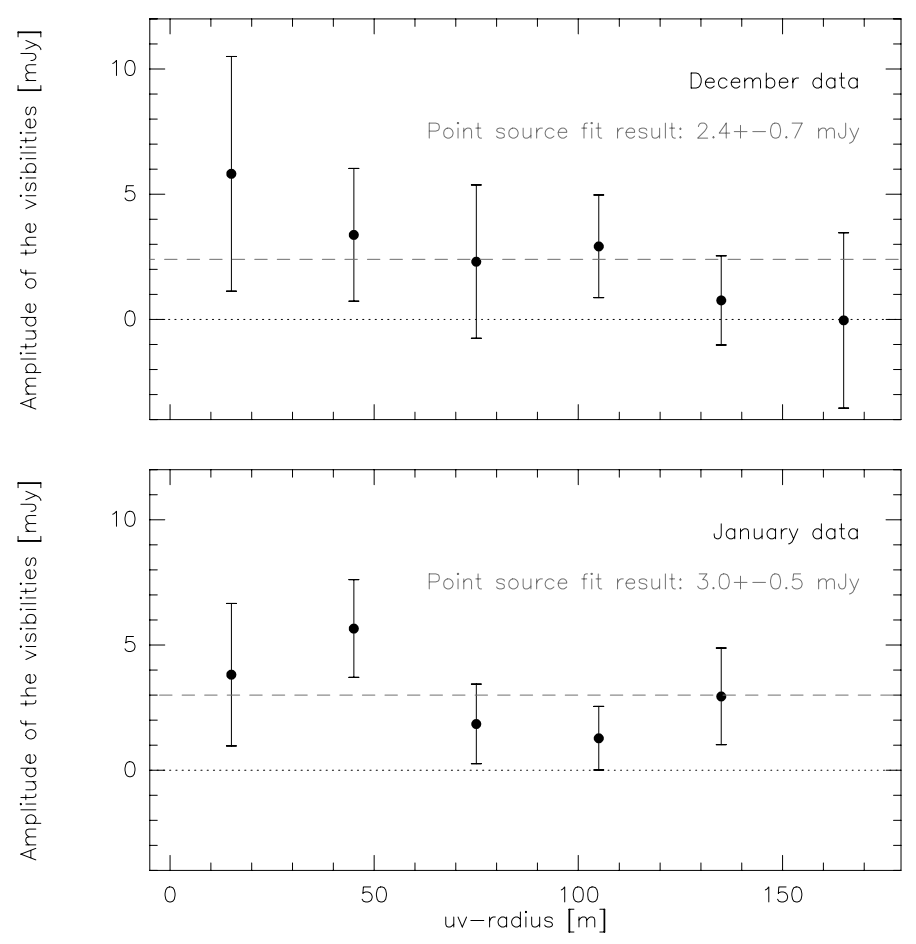

Fig. 1. Real part of the visibilities as a function of uv-radius for the December (top) and January data (bottom). The visibility tables have been shifted to the position found by the fitting procedure so that their real part represents the point source flux. To increase the signal-to-noise ratio for individual points, the visibilities have been averaged in $30 \mathrm{~m}$ bins of uv-radius. The result of the fitting procedure in the uv-plane is presented by the dashed, grey line.

were $0235+164$ and $0048-097$ and 3C 454.3 was used as a reference to determine the absolute flux scale.

Fitting the FT of a point source to the observed visibilities in the Fourier plane, we retrieve a flux of $3.0 \pm 0.5$ mJy located at an offset of $\left(1.4^{\prime \prime},-0.1^{\prime \prime}\right)$ in (RA, Dec) with respect to the pointed position (the astrometric precision is $\left.\sim 0.2^{\prime \prime}\right)$. Compared to the latest solution (JPL K074/27), the offset is $\left(1.8^{\prime \prime}, 1.2^{\prime \prime}\right)$. This result is illustrated with the real part of the visibilities presented as a function of the uv-radius in Fig. 1. Taking into account the flux calibration uncertainty, we obtain a flux of $3.0_{-1.0}^{+1.2} \mathrm{mJy}(1 \sigma)$ or $3.0_{-1.8}^{+2.4} \mathrm{mJy}(3 \sigma)$. In the following analysis, we use only the flux measured on January 8, as it offers a higher signal-to-noise ratio.

We assume that the detected emission is due solely to the nucleus thermal emission. The contribution of dust thermal emission is expected to be weak, based on previous observations of the dust continuum in the millimetre and submillimetre domains (e.g. Jewitt \& Luu 1992; Jewitt \& Matthews 1997). For example, using the JCMT (HPBW = 19") Jewitt \& Matthews (1997) measured a flux of $6.4 \mathrm{mJy}$ at $350 \mu \mathrm{m}$ for comet C/1996 B2 (Hyakutake) at $r_{\mathrm{h}}=1.08 \mathrm{AU}$ and $\Delta=0.12 \mathrm{AU}$. Assuming that the dust opacity varies according to $\lambda^{-0.89}$ (Jewitt \& Matthews 1997) and that the dust brightness distribution varies according to $\rho^{-1}$, where $\rho$ is the distance to the nucleus, we derive a dust flux at $240 \mathrm{GHz}$ of $0.05 \mathrm{mJy}$ for a beam size of $1^{\prime \prime}$ and a geocentric distance of $0.26 \mathrm{AU}$. The gaseous activity of $8 \mathrm{P} /$ Tuttle in early January 2008 was typically 5-10 times lower than the activity of Hyakutake at $r_{\mathrm{h}}=1.08$ AU (Mumma et al. 1996; Bonev et al. 2008; Kobayashi et al. 2010). Hence, the contribution of the dust emission to the detected flux at $240 \mathrm{GHz}$ in comet $8 \mathrm{P} /$ Tuttle should not exceed $0.01 \mathrm{mJy}$, compared to the $3.0 \mathrm{mJy}$ of the nucleus.

\section{Nucleus thermal model}

\subsection{Shape model}

Harmon et al. $(2008,2010)$ interpreted their Arecibo radar observations of the nucleus of comet $8 \mathrm{P} /$ Tuttle as implying a contact binary and proposed a shape model composed of two spheroids in contact. Lamy et al. (in prep.) found that the light curve of the nucleus derived from their Hubble Space Telescope observations was indeed most accurately explained by a binary configuration and derived a model composed of two spheres in contact. However, these two models profoundly differ first in the direction of the rotational axis and second, in the size ratio of the two components of the nucleus. Groussin et al. (in prep.) used both shape models to interpret Spitzer Space Telescope thermal light curves of the nucleus and concluded that the shape model of Lamy et al. (in prep.) most closely matches the SST observations.

The shape model of Lamy et al. (in prep.) consists of two contact spheres with respective radii of $2.6 \pm 0.1 \mathrm{~km}$ and $1.1 \pm 0.1 \mathrm{~km}$; a sphere with a radius of $2.8 \mathrm{~km}$ would have the same cross-section. The pole orientation is $\mathrm{RA}=285^{\circ}$ and Dec $=+20^{\circ}$, which gives an aspect angle (defined as the angle between the spin vector and the comet-Earth vector) of $82^{\circ}$ on 29 Dec. 2007 and $103^{\circ}$ on 08 Jan. 2008, close to an equatorial view. The rotational period is $11.4 \mathrm{~h}$ (Lamy et al. 2008) in this model. Harmon et al. (2010) derived a more precise value of the period based on the radar experiments carried out at Arecibo: $11.385 \pm 0.004 \mathrm{~h}$. By rotating the nucleus back from the radar epoch to the HST epoch, we were able to connect the rotation phases and thus determine the true or sidereal rotation period $P_{\text {sid }}=11.444 \pm 0.001 \mathrm{~h}$ (see Lamy et al., in prep.).

In view of the above discussion, we used two different shape models for our analysis: i) a simple spherical shape model with a radius of $2.8 \mathrm{~km}$ and ii) the more complex and realistic shape model of Lamy et al. (in prep.), which consists of two contact spheres. In the later model, the shape is divided into 2560 triangular facets of comparable size. We consider the size of these two shape models as robust, so that it is a fixed parameter. 


\subsection{Thermal model}

The interpretation of the millimetric observations requires a thermal model for the nucleus. We used the thermal model presented in Groussin et al. (in prep.) for comet 8P/Tuttle, which was extensively described in several previous articles (e.g., Groussin et al. 2004; Lamy et al. 2010). For each facet of the shape model, we solve for the surface energy balance between the flux received from the Sun, the re-radiated flux, and the heat conduction into the nucleus. As the nucleus rotates around its spin axis, the illumination changes, and the heat conduction equation is computed for each facet considering a one-dimensional timedependent equation. The projected shadows are taken into account in our model. As a result, we obtained the temperature of each facet as a function of time, over one rotation period. We then integrated the flux over each facet to calculate the total millimetric flux received by the observer as a function of time and wavelength $(1.25 \mathrm{~mm})$.

The active area of $8 \mathrm{P} /$ Tuttle is restricted to $<15 \%$ of the surface (Groussin et al., in prep.). As for 9P/Tempel 1 with an active area of $9 \%$ (Lisse et al. 2005), the sublimation of water ice can be neglected in the energy balance for the calculation of the thermal flux emitted from the nucleus surface (Groussin et al. 2007).

\section{Results}

We use the above simple spherical shape model with a radius of $2.8 \mathrm{~km}$ and our thermal model with the parameters of Groussin et al. (2008) (thermal inertia $I=0 \mathrm{~J} \mathrm{~K}^{-1} \mathrm{~m}^{-2} \mathrm{~s}^{-1 / 2}$, beaming factor $\eta=0.7$, emissivity $\epsilon=0.95$ ), which is in that case identical to the Standard Thermal Model (Lebofsky \& Spencer 1989), to infer a millimetric flux of $5.6 \mathrm{mJy}$ at $1.25 \mathrm{~mm}$ on 8 Jan. 2008 . This is higher than, though barely in agreement with, our observed flux of $3.0_{-1.0}^{+1.2} \mathrm{mJy}(1 \sigma)$ or $3.0_{-1.8}^{+2.4} \mathrm{mJy}(3 \sigma)$, which corresponds to a smaller radius of $r=2.0 \pm 0.4 \mathrm{~km}(1 \sigma)$ using the same thermal model and parameters. To investigate the origin of this discrepancy, we studied the influence of several parameters in the calculation of the flux at $1.25 \mathrm{~mm}$ : i) shape; ii) thermal inertia $I$; iii) beaming factor $\eta$; and iv) emissivity $\epsilon$ from the surface and sub-surface layers. Our goal is to investigate which of these parameters can help to decrease the nucleus flux to make it compatible with our observations.

\subsection{Shape effect}

The simple calculation described above has been made using our spherical shape model with a radius of $2.8 \mathrm{~km}$. As described in Sect. 3, a more realistic shape model exists, composed of two contact spheres (Lamy et al. 2008; Lamy et al., in prep.). With this shape model, the flux is not constant with time as for the spherical case, but changes during the nucleus rotation to produce a thermal lightcurve. Under our standard assumptions ( $I=0, \eta=0.7, \epsilon=0.95)$, we calculated the synthetic thermal light curve of this shape model, as illustrated in Fig. 2. The flux varies with time, with a minimum of $\sim 4.45 \mathrm{mJy}$ and a maximum of $\sim 5.65 \mathrm{mJy}$. The maximum flux is reached when the primary and secondary are both illuminated, which is equivalent to a spherical nucleus of radius $2.8 \mathrm{~km}$ as explained in Sect. 3.1. The minimum flux is reached when the secondary eclipses the primary, which is equivalent to a spherical nucleus of radius $2.5 \mathrm{~km}$. As the amplitude of the ligth curve is large $(1.2 \mathrm{mJy})$, depending on the time of observation, the observed flux can be quite different.

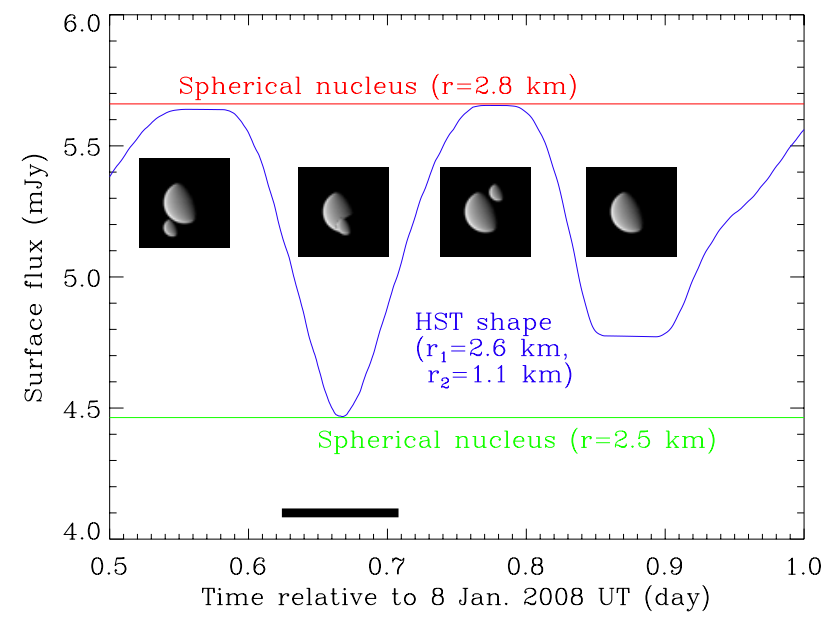

Fig. 2. Thermal lightcurve of $8 \mathrm{P} /$ Tuttle as seen from the Plateau de Bure on 8 January 2008. We used our standard set of parameters for the thermal inertia $(I=0)$, the beaming factor $(\eta=0.7)$ and the emissivity $(\epsilon=0.95)$. The shape model is that of 8P/Tuttle derived from Hubble Space Telescope observations (Lamy et al. 2008; Lamy et al., in prep.). The lightcurve has been phased relatively to the radar observations of Harmon et al. (2010), performed $\sim 10$ rotation periods earlier. The maximum (resp. minimum) flux corresponds to the emission of a spherical nucleus of $2.8 \mathrm{~km}$ (resp. $2.5 \mathrm{~km}$ ). The horizontal thick solid line corresponds to the observation time at Plateau de Bure ( $2 \mathrm{~h}$ long).

Using the sidereal rotation period of $11.444 \pm 0.001 \mathrm{~h}$, we phased the observations performed at Plateau de Bure on 8 Jan. 2008 with the Arecibo radar experiments of Harmon et al. (2010) performed in early January 2008. The reference epoch of the Arecibo radar study is Jan. 4.0046, i.e. $\sim 10$ rotation periods earlier than our observations, which translates into an uncertainty of 0.0004 day, i.e. smaller than $1 \mathrm{~min}$. At the reference epoch, the rotation phase is $-5 \pm 1.5^{\circ}$ with $0^{\circ}$ being the time when the nucleus is seen broadside, with the larger lobe approaching (Harmon et al. 2010) and corresponds to the maximum that follows the lightcurve minimum in Fig. 2, on Jan. 8.75. The $1.5^{\circ}$ error bar in phase at the reference epoch adds another 3 min of uncertainty to the rephasing. The total uncertainty does not exceed 5 min and is small compared to the length of the Plateau de Bure observations ( $2 \mathrm{~h}$ ).

The lightcurve presented in Fig. 2 has been rephased and one can see that the Plateau de Bure observations (around Jan. 8.67) were performed around its minimum. Under the standard parameters, we expect a flux of $\sim 4.45 \mathrm{mJy}$ at that time. This is still higher than the $3.0_{-1.0}^{+1.2} \mathrm{mJy}$ measured with the Plateau de Bure, thus we considered whether a different set of thermal parameters for the nucleus can explain this discrepancy.

\subsection{Thermal inertia effect}

We present in Fig. 3 the thermal lightcurve expected from the comet 8P/Tuttle for different values of the nucleus thermal inertia. As the thermal inertia increases, the diurnal temperature variations decrease: the temperatures become cooler on the day side and warmer on the night side. In our case, since the phase angle is large $\left(65^{\circ}\right)$, the heating on the night side is more pronounced than the cooling on the day side, and overall, the observed flux becomes larger as thermal inertia increases. It is clear from Fig. 3 that a larger thermal inertia does not help us 


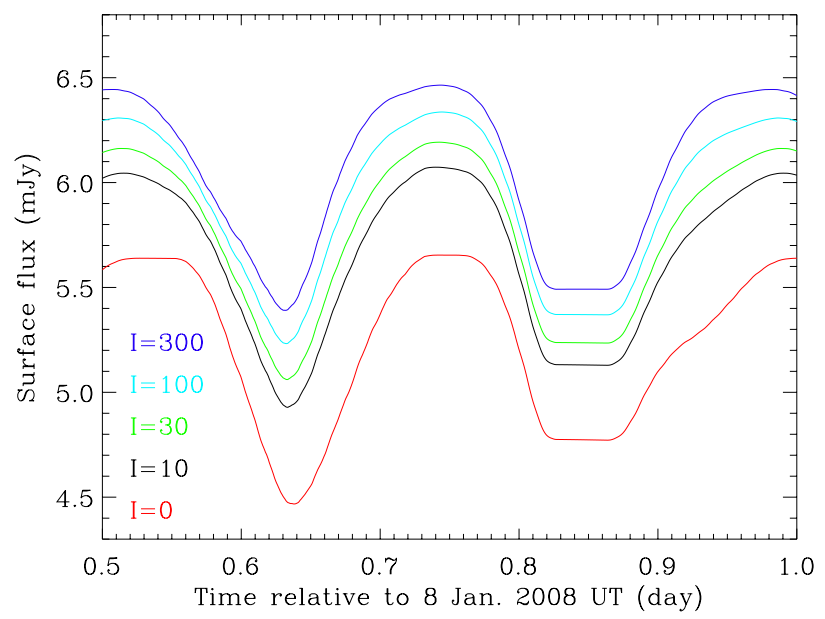

Fig. 3. Expected thermal lightcurves of the comet $8 \mathrm{P} /$ Tuttle at $1.25 \mathrm{~mm}$ for different values of the nucleus thermal inertia between 0 (in red) and 300 MKS (in blue). The beaming factor is $\eta=0.7$ and the emissivity $\epsilon=0.95$. The geometrical configuration is that of the observations of 8P/Tuttle on 08 January $2008\left(r_{\mathrm{h}}=1.07 \mathrm{AU}, \Delta=0.28 \mathrm{AU}\right.$, phase angle $\left.\phi=65^{\circ}\right)$.

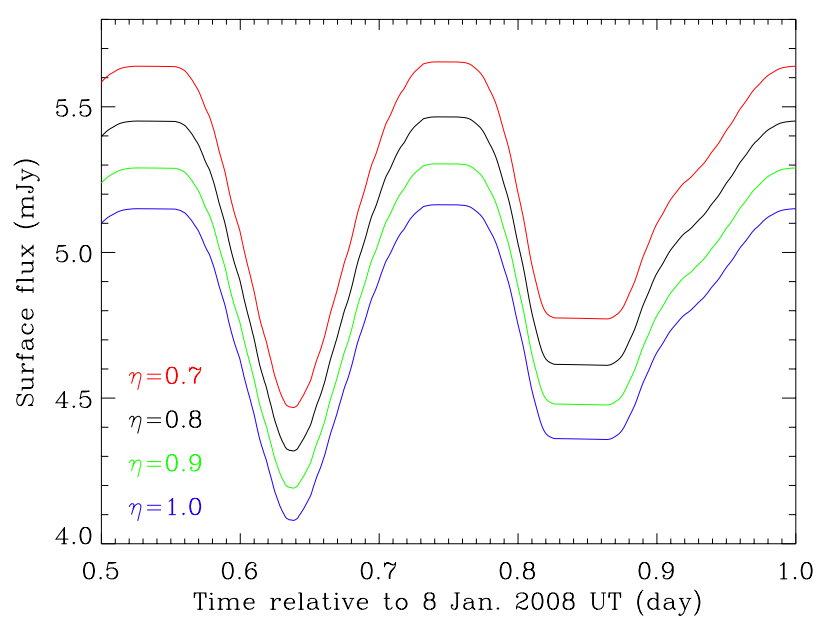

Fig. 4. Expected thermal lightcurves of the comet 8P/Tuttle at $1.25 \mathrm{~mm}$ for different values of the surface beaming factor $(\eta)$. The thermal inertia is $I=0$ and the emissivity $\epsilon=0.95$. The geometrical configuration is identical to that of the observations of 8P/Tuttle in January 2008.

to solve this problem as the flux then only gets larger. In the range $0-200 \mathrm{~J} \mathrm{~K}^{-1} \mathrm{~m}^{-2} \mathrm{~s}^{-1 / 2}$ for the thermal inertia estimated by Groussin et al. (2008), we then favour the lower values (typically $\leq 10 \mathrm{~J} \mathrm{~K}^{-1} \mathrm{~m}^{-2} \mathrm{~s}^{-1 / 2}$ ).

\subsection{Beaming factor effect}

We present in Fig. 4 the thermal lightcurve emitted by our synthesized, bilobate nucleus as a function of the beaming factor $\eta$. In our thermal model, the beaming factor $(\eta)$ follows the definition given by Lagerros (1998), which only reflects the influence of the surface roughness and $\eta$ has to be lower or equal to 1.0. In addition, according to Lagerros (1998), $\eta$ must be larger than 0.7 to avoid unrealistic roughness, with rms slopes exceeding $45^{\circ}$. The acceptable range for $\eta$ is then $0.7-1.0$, as illustrated in Fig. 4 . When roughness increases (i.e., $\eta$ decreases), the

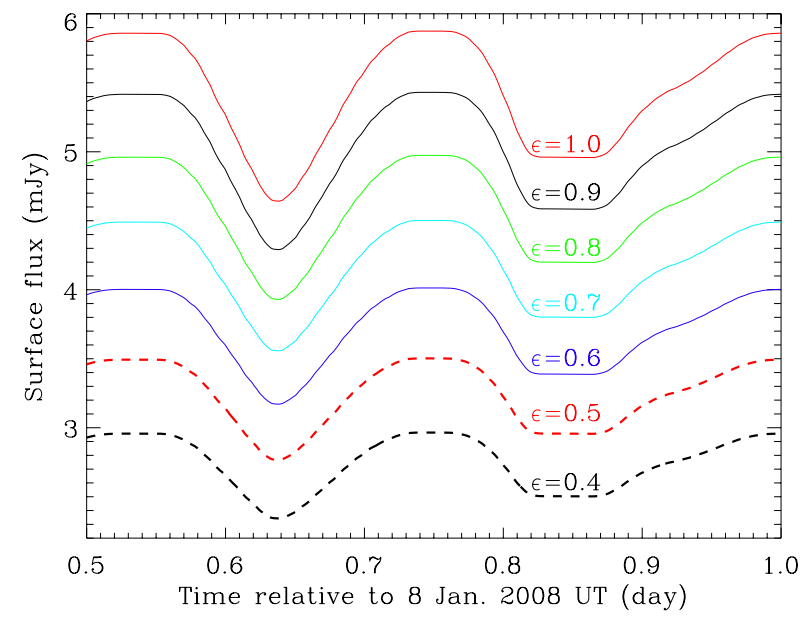

Fig. 5. Expected thermal lightcurves of the comet $8 \mathrm{P} /$ Tuttle at $1.25 \mathrm{~mm}$ for different values of the surface emissivity $\epsilon$ ranging from 1 (highest, solid red line) to 0.4 (lowest, dashed yellow line). The thermal inertia is $I=0$ and the beaming factor $\eta=0.7$. The geometrical configuration is identical to that of the observations of 8P/Tuttle in January 2008.

surface temperature increases owing to self-heating on the surface, and the total flux increases. Changing $\eta$ from 0.7 (Groussin et al. 2008) to 1.0 decreases the minimum flux from $4.45 \mathrm{mJy}$ to $4.1 \mathrm{mJy}$, which is a small improvement but an insufficient one for explaining the observed flux of $3.0 \mathrm{mJy}$.

Groussin et al. (2008) used SST observations of comet $8 \mathrm{P} /$ Tuttle to derive a beaming factor $\eta$ of the order of 0.7 . This value is constrained by the infrared spectrograph (IRS) observations, which lasted only $10 \mathrm{~min}$, a short time compared to the rotation period of $\sim 11.4 \mathrm{~h}$. The SST observations were performed on 2.76 Nov. 2007. The nucleus was in the same rotation phase 141 periods later, on $8.65 \pm 0.02 \mathrm{Jan}$. 2008, which coincides with the time the Plateau de Bure observations (Fig. 2). As a result, although higher $\eta$ values correspond to lower millimetre fluxes, we favour the $\eta=0.7$, which is well constrained by the Spitzer observations.

Delbó et al. (2003) proposed that there is an increase of the beaming factor with phase angle for near-earth asteroids (NEAs). If confirmed, this trend could explain why our observations performed at a $65^{\circ}$ phase angle favour a larger value of $\eta$ close to one, while SST observations performed at a $39^{\circ}$ phase angle favour a smaller value, close to 0.7 . However, according to Delbó et al. (2003), more observations are required to confirm this trend.

Finally, it cannot be excluded that the effect of the roughness $(\eta)$ may be wavelength dependent. Roughness on a micron scale could indeed be more important than on a millimetre scale, resulting in a smaller beaming factor in the infrared. However, this point cannot be confirmed since we cannot rule out there being a fractal surface, in which case the roughness would be more or less scale-independent.

\subsection{Emissivity and depth effect}

The thermal flux emitted from the nucleus is proportional to the surface emissivity, as illustrated in Fig. 5. The flux at the lightcurve minimum is in $1 \sigma$ agreement with our observations for an emissivity lower than 0.8 . While the surface emissivity of small bodies in the mid-infrared is close to unity, it is still 
unknown at millimetre wavelengths and might be lower than in the mid-infrared. This point has already been addressed by Fernández (2002) for comet C/1995 O1 Hale-Bopp, where an emissivity of 0.5 is required in the millimetic range to reconcile the nucleus sizes derived from infrared and millimetric observations. On the basis of asteroid observations in the sub-millimetre, Redman et al. (1998) found that the emissivity of seven asteroids decreases with increasing wavelength. The observations of asteroid (2867) Steins at 1.6 and $0.53 \mathrm{~mm}$ performed with the radiometer MIRO onboard the Rosetta spacecraft confirm this trend: Gulkis et al. (2010) found an emissivity decreasing from $0.85-9$ at 1.6 to $0.6-0.7$ at $0.53 \mathrm{~mm}$. A low millimetric emissivity of 0.6 was also found for asteroid 4 Vesta by Mueller \& Lagerros (1998). Overall, this means that our values $(\epsilon<0.8)$ are plausible.

A possible physical explanation of the lower emissivity is that part of the millimetre thermal flux arises from sub-surface layers that are colder than the surface itself. This temperature gradient is expected given the low thermal inertia of the nucleus. The depth of the main contributing layer depends on the material opacity, which is unknown. Measurements for rocks showed that in most materials this depth lies between 3 and $100 \lambda$ (Campbell $\&$ Ulrichs 1969). A depth of $10 \lambda$ is commonly used when dealing with planetary surfaces (e.g. $11 \lambda$ is used for the martian surface regolith, Muhleman \& Berge 1991; Goldin et al. 1997). Depending on the upper-layer temperature gradient (controlled by the thermal inertia) and the relative contribution of the different layers to the overall emission, the resulting spectral emissivity of the surface can be significantly lower than the usual infrared value close to unity.

To investigate this effect, we calculated the nucleus integrated flux as a function of depth (down to $20 \mathrm{~cm}$ ), relative to the surface flux, for different thermal inertia values. Figure 6 illustrates our results: the contribution of the first sub-surface layers is comparable or larger to the surface contribution itself because we are sensitive to the emission of the afternoon side of the nucleus where the temperature is still warm, even in the shadowed area. At a certain depth (which depends on the thermal inertia) the contribution of the sub-surface layers sharply declines as the diurnal temperature variations become negligible.

We use a Poissonian weighted function (that peaks at 10 $\lambda$ ) to describe the relative contributions of the sub-surface layers, which seems to be a good approximation of the work of Moullet (2008), and we integrate the weighted flux from Fig. 6 as a function of depth, to derive the effective outcoming flux with respect to a pure surface emission. The results are presented in Table 2. Depending on the thermal inertia, the flux is reduced by $9 \%$ to $17 \%$ compared to a pure surface emission. A low thermal inertia $\left(\leq 10 \mathrm{~J} \mathrm{~K}^{-1} \mathrm{~m}^{-2} \mathrm{~s}^{-1 / 2}\right)$ is in better agreement with our observations as explained in Sect. 4.2, and the flux is then reduced by $14-17 \%$, which corresponds to an "effective" emissivity of 0.79-0.82 assuming our standard surface emissivity of 0.95 . According to Fig. 5 and as explained above, this allow us to reproduce our observations to within $1 \sigma$.

Our results confirm that a low emissivity in the millimetric wavelength range can result from the emission of sub-surface layers with a low thermal inertia. At mid-infrared wavelengths of typically around $10 \mu \mathrm{m}, 10 \lambda$ corresponds to $0.1 \mathrm{~mm}$, a depth over which the flux is close the surface flux (Fig. 6), explaining why the above effect only matters in the millimetric wavelength range and beyond, in the centimetric and radar wavelength range. However, for the centimetric and radar wavelengths, there exists a few notable exceptions, in particular Ganymede, whose centrimetric emission corresponds to a brightness temperature

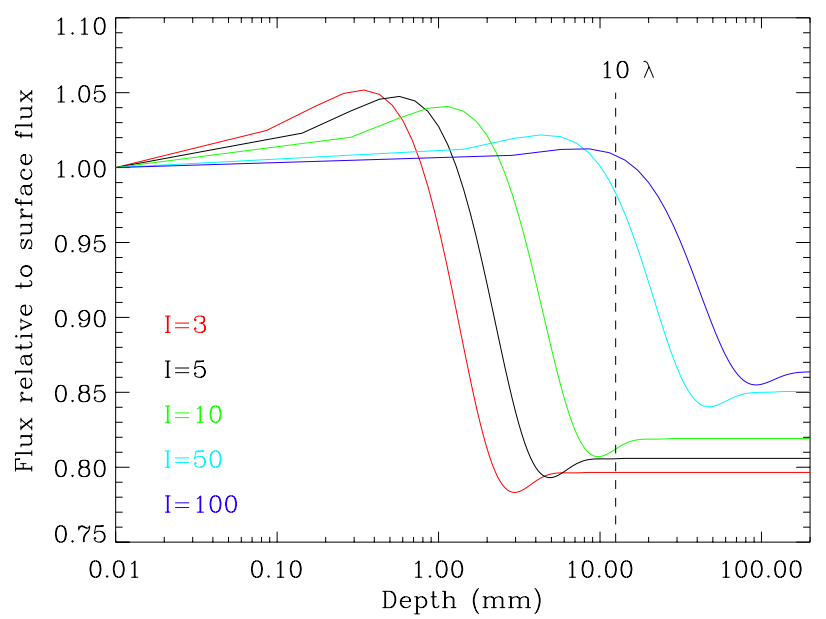

Fig. 6. Thermal emission from a sub-surface layer (relative to surface emission) as a function of its depth and for different values of the thermal inertia.

Table 2. Nucleus thermal flux integrated over depth, compared to pure surface emission (1.0).

\begin{tabular}{lc}
\hline \hline $\begin{array}{l}\text { Thermal inertia } \\
\mathrm{J} \mathrm{K}^{-1} \mathrm{~m}^{-2} \mathrm{~s}^{-1 / 2}\end{array}$ & Relative depth-integrated flux \\
\hline 3 & 0.83 \\
5 & 0.84 \\
10 & 0.86 \\
50 & 0.88 \\
100 & 0.91 \\
\hline
\end{tabular}

of about 55-88 K, below any acceptable sub-surface temperature for this body (Muhleman \& Berge 1991), and whose radar reflection is highly anomalous (Ostro \& Shoemaker 1990). In this case, the satellite must also have a higher reflectivity which is probably caused by backscattering from the surface.

\section{Summary}

We have reported the first detection of the thermal emission of a cometary nucleus at millimetre wavelengths since C/1995 O1 Hale-Bopp, performed at Plateau de Bure on 8 Jan. 2008. Using the rotation period derived by Harmon et al. (2010), we have phased our observations with respect to their radar experiments and found that Plateau de Bure observations were performed at a minimum of the lightcurve. We used the shape model developed by Lamy et al. (in prep.) for 8P/Tuttle nucleus to compute thermal emission lightcurves using different thermal parameter sets. From our analysis, we can conclude that to match our observed flux of $3.0_{-1.0}^{+1.2} \mathrm{mJy}$ at $1.25 \mathrm{~mm}$, the thermal inertia of the nucleus should be low, typically $\leq 10 \mathrm{~J} \mathrm{~K}^{-1} \mathrm{~m}^{-2} \mathrm{~s}^{-1 / 2}$, in agreement with the range $0-200 \mathrm{~J} \mathrm{~K}^{-1} \mathrm{~m}^{-2} \mathrm{~s}^{-1 / 2}$ derived from Spitzer observations (Groussin et al. 2008). In addition, the millimetric emissivity of the nucleus should be lower than 0.8. According to our thermal model, such a low value can be due to the non-negligible emission of the sub-surface layers of the nucleus, which are colder than the surface at depths of a few millimetre or more for low thermal inertia values. Similar and even lower values of the millimetric emissivity have already been proposed to explain the low flux emitted by asteroids 
J. Boissier et al.: Earth-based detection of the millimetric thermal emission from the nucleus of comet 8P/Tuttle

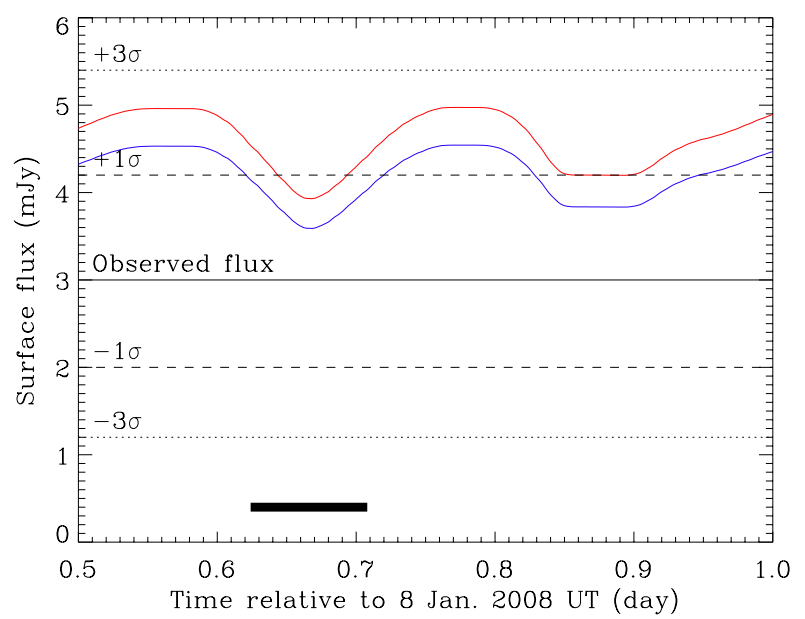

Fig. 7. Same figure as Fig. 2 but for $I=0, \epsilon=0.8, \eta=0.7$ (red curve) or $\eta=1.0$ (blue curve). For $\eta=1.0$, which is our preferred value, about half of the thermal lightcurve agrees with our observations at the $1 \sigma$ level.

(Redman et al. 1998; Mueller \& Lagerros 1998; Fernández 2002). As for the beaming factor, the lowest flux of the lightucurve is in $1 \sigma$ agreement with our observed flux for any value in the plausible range $0.7-1.0$, there being closer agreement towards the higher edge. However, we favour $\eta=0.7$, which is the only value in agreement with Spitzer IRS observations (Groussin et al., in prep.). From the above conclusions, we have generated a synthetic lightcurve for the nucleus of comet 8P/Tuttle, as observed from the Plateau de Bure on 8 Jan. 2008, using the parameters $I=0, \epsilon=0.8$, and $\eta$ in the range $0.7-1.0$ to cover the possible values. The result is illustrated in Fig. 7.

In the future, the Rosetta spacecraft, equipped with a microwave instrument operating at 190 and $562 \mathrm{GHz}$ (Gulkis et al. 2007), will provide an unprecedented dataset to study the surface of a cometary nucleus and its thermal properties. It will also be important to repeat ground-based studies of other comets, to determine whether all comets have similar properties or if they change from one comet to another, which would be indicative of different surface natures. The (sub-)millimetre interferometer ALMA will offer a significant gain in sensitivity and allow similar studies of a larger sample of comets, from both the ecliptic and the Oort cloud dynamical classes.
Acknowledgements. The research leading to these results has received funding from the European Community's Seventh Framework Programme (FP7/20072013) under grant agreement No. 229517.

\section{References}

Altenhoff, W. J., Bieging, J. H., Butler, B., et al. 1999, A\&A, 348, 1020 Biver, N., Lis, D. C., Fray, N., et al. 2008, LPI Contributions, 1405, 8151 Bonev, B. P., Mumma, M. J., Radeva, Y. L., et al. 2008, ApJ, 680, L61 Boss, A. P. 2003, ApJ, 599, 577

Cameron, A. G. W. 1978, M\&P, 18, 5

Campbell, M. J., \& Ulrichs, J. 1969, J. Geophys. Res., 74, 5867

Crovisier, J., Biver, N., Bockelée-Morvan, D., et al. 2009, EM\&P, 105, 267

Delbó, M., Harris, A. W., Binzel, R. P., Pravec, P., \& Davies, J. K. 2003, Icarus, 166,116

Duncan, M., Quinn, T., \& Tremaine, S. 1988, ApJ, 328, L69

Fernández, Y. R. 2002, EM\&P, 89, 3

Goldin, A. B., Kowitt, M. S., Cheng, E. S., et al. 1997, ApJ, 488, L161

Groussin, O., Lamy, P., \& Jorda, L. 2004, A\&A, 413, 1163

Groussin, O., A'Hearn, M. F., Li, J.-Y., et al. 2007, Icarus, 187, 16

Groussin, O., Kelley, M., Lamy, P., et al. 2008, BAAS, 40, 393

Gulkis, S., Allen, M., Backus, C., et al. 2007, P\&SS, 55, 1050

Gulkis, S., Keihm, S., Kamp, L., et al. 2010, P\&SS, 58, 1077

Harmon, J. K., Nolan, M. C., Howell, E. S., \& Giorgini, J. D. 2008, LPI Contributions, 1405, 8025

Harmon, J. K., Nolan, M. C., Giorgini, J. D., \& Howell, E. S. 2010, Icarus, 207, 499

Jewitt, D., \& Luu, J. 1992, Icarus, 100, 187

Jewitt, D. C., \& Matthews, H. E. 1997, ApJ, 113, 1145

Kobayashi, H., Bockelée-Morvan, D., Kawakita, H., et al. 2010, A\&A, 509, A80 Lagerros, J. S. V. 1998, A\&A, 332, 1123

Lamy, P. L., Toth, I., Fernandez, Y. R., \& Weaver, H. A. 2004, Comets II, ed. M. C. Festou, H. U. Keller, \& H. A. Weaver, 223

Lamy, P. L., Toth, I., Jorda, L., et al. 2008, BAAS, 40, 393

Lamy, P., Groussin, O., Fornasier, S., et al. 2010, A\&A, 516, A74

Lebofsky, L. A., \& Spencer, J. R. 1989, in Asteroids II, ed. R. P. Binzel, T. Gehrels, \& M. S. Matthews, 128

Licandro, J., Tancredi, G., Lindgren, M., Rickman, H., \& Hutton, R. G. 2000, Icarus, 147, 161

Lisse, C. M., A'Hearn, M. F., Groussin, O., et al. 2005, ApJ, 625, L139

Moullet, A. 2008, Ph.D., Thesis, Thèse de doctorat de l'Université Paris 7 Diderot

Mueller, T. G., \& Lagerros, J. S. V. 1998, A\&A, 338, 340

Muhleman, D. O., \& Berge, G. L. 1991, Icarus, 92, 263

Mumma, M. J., Disanti, M. A., Dello Russo, N., et al. 1996, Science, 272, 1310

Ostro, S. J., \& Shoemaker, E. M. 1990, Icarus, 85, 335

Pety, J. 2005, in SF2A-2005: Semaine de l'Astrophysique Française, ed. F. Casoli, T. Contini, J. M. Hameury, \& L. Pagani, 721

Pollack, J. B., Hubickyj, O., Bodenheimer, P., et al. 1996, Icarus, 124, 62

Redman, R. O., Feldman, P. A., \& Matthews, H. E. 1998, ApJ, 116, 1478

Safronov, V. S., \& Zvjagina, E. V. 1969, Icarus, 10, 109 